\title{
STUDY OF DOG PLATELETS INFECTED WITH EHRLICHIA PLATYS USING SCANNING ELECTRON MICROSCOPY.
}

\author{
Maldonado, $\mathbf{N}^{*}$; Palmar, $\mathbf{M}^{*}$; Hernández, J P*; Alvarado -Arraga, C**; Parra \\ O**. \\ *Instituto de Investigaciones Biológicas, Facultad de Medicina-LUZ; **Unidad de \\ Investigaciones Clínicas, Facultad de Cs. Veterinarias- Universidad del Zulia \\ e-mail : naudyjaneth@hotmail.com
}

The studies on three - dimensional structure of healthy animal blood platelets are scarce, and the three - dimensional characteristics of blood platelets infected by some type of external agent that causes disease have not been reported up to now. The goal of the present research was the determination of the three - dimensional structure of platelets infected with Ehrlichia platys, which has been diagnosed in platelets from dogs of Maracaibo - Venezuela, using light and transmission electron microscopy techniques. Blood samples were taken from dogs with symptoms of ehrlichiosis and healthy dogs, given by Veterinary Sciences Faculty of University of Zulia. Each one of these samples was centrifuged to obtain platelet - rich plasma (PRP). A drop of this PRP was pipetted directly onto a circular cover glass and glutaraldehyde $3 \%$ was added. After one hour, the sample was processed following the scanning electron microscopy (SEM) conventional technique and observed in a SEM Phillips XL 30. Infected platelets were pleomorphic and were 4.3 to $5.4 \mu \mathrm{m}$ in diameter, with projections emerging from their external surface. Platelets displayed 7 to 13 short projections, with blunt aspect in their ends (figure 1), which were 0.2 to 0.8 of $\mu \mathrm{m}$ in length and located throughout the platelet surface. Some platelets displayed small protuberances on their surface (figure 2 ), whereas others only showed certain convolutions. Larger pseudopods than the rest of the surface projections were also distinguished (figure 3). Small apertures were randomly visualized in platelet membrane. A slight roughness in the membrane was demonstrated. These findings demonstrate an alteration of the platelet surface when it is infected by a bacteriological agent that causes a disease. 


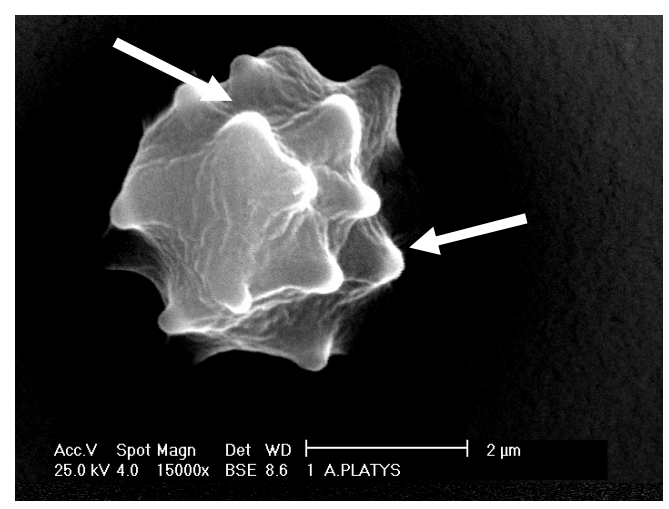

Fig. 1 : Scanning electron micrograph from dog blood platelet infected with Ehrlichia platys (A. platys) exhibiting in its surface short projections with blunt or blunted aspect (arrows).

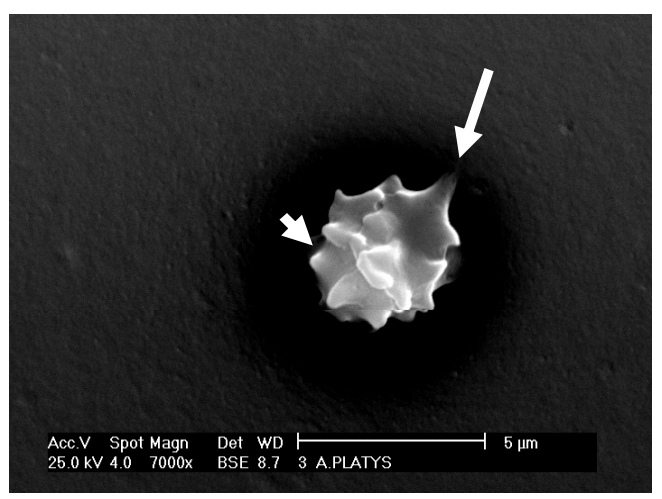

Fig. 3 : Scanning electron micrograph from dog blood platelet infected with Ehrlichia platys (A. platys) in which are visualized larger pseudopods (arrow head) than the rest of the surface projections (arrow)

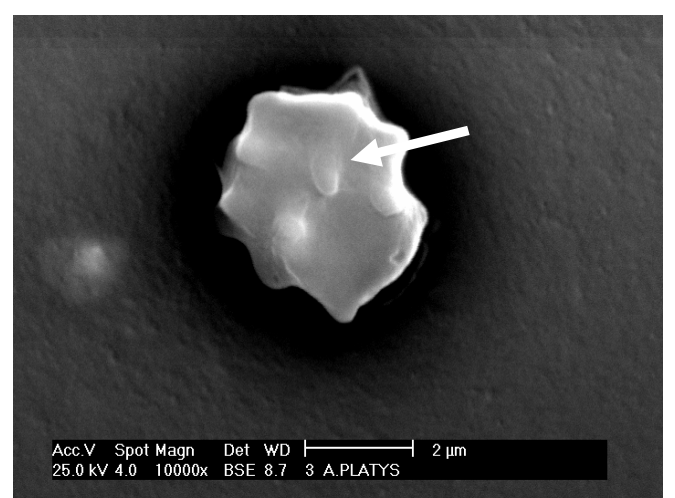

Fig. 2 : Scanning electron micrograph from dog blood platelet infected with Ehrlichia platys (A. platys) in which are observed small protuberances displayed on their surface (arrow).

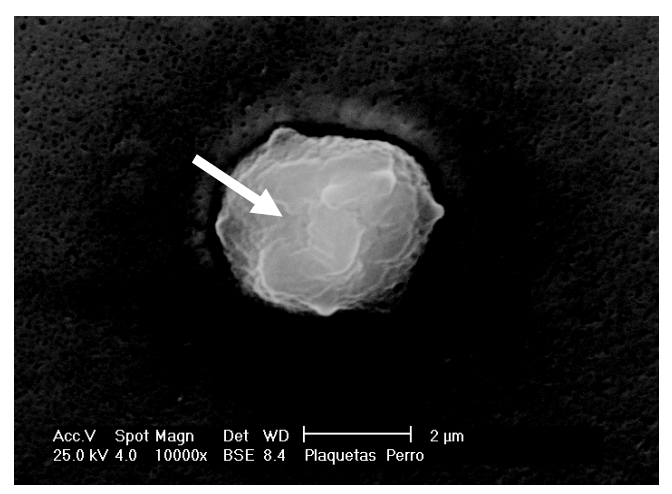

Fig. 4 : Scanning electron micrograph from healthy dog blood platelet in which the rough aspect of its surface is distinguished (arrow). 